\title{
The detection of pentobarbital in euthanized domestic dogs
}

\author{
Jessica A Hvozdovich? MS, Jason H Byrd PhD DABFE, Lisa L Farina² DVM DACVP, Bruce A Goldberger ${ }^{1}$ PhD FABFT
}

\begin{abstract}
This preliminary study examined the postmortem distribution of pentobarbital in the remains of three domestic dogs (Canis lupus familiaris) after intravenous administration of pentobarbital sodium to determine optimal sampling practices for veterinary forensic casework. It was thought that pentobarbital would be absent in many traditional toxicological samples, such as bile and urine, because of the brief period between administration and death. However, all samples tested via gas chromatography, coupled with a nitrogen-phosphorus detector, contained quantifiable levels of pentobarbital. These initial results suggest that when the Florida Animal Control Association's recommended method of euthanasia is used in healthy individuals, pentobarbital extensively distributes throughout the body.
\end{abstract}

KEYWORDS: veterinary forensic sciences, veterinary pathology, toxicology, pentobarbital, GC-NPD, euthanasia

Remains at varying stages of decomposition are often recovered on animal crime scenes. Informed sampling decisions are critical for toxicological analysis to ensure that reliable results are obtained and used to assist in cause-of-death determinations. With suspected chemical euthanasia, false negatives are concerning. It is unknown if the active components of euthanasia solutions spend enough time in circulation with standard administration practices to permeate throughout the body and deposit in traditional toxicological matrices at detectable quantities. Previous studies examining pentobarbital distribution have involved protocols that could affect drug distribution such as coadministration of other compounds (Watterson et al. 2010), the use of intracardiac injections, sacrifice via carbon dioxide (Desrosier and Watterson 2010), the use of pentobarbital for both sedation and euthanasia (Wyman et al. 2011), and oral dosing of euthanasia solution (Winek et al. 1985). In order to obtain preliminary data regarding pentobarbital distribution with standard veterinary practices, samples collected from three humanely euthanized domestic dogs were analyzed using gas chromatography coupled with a nitrogen-phosphorus detector (GC-NPD). This initial investigation was performed to determine which specimen types produced the most reliable results for drug detection and warrant further investigation under different conditions.

\section{Materials and Methods}

The research subjects (Canis lupus familiaris) were selected based on age (young, adult; 1-6 years), size (>15 kg), and health status (no known diseases) to ensure that adequate sample quantities were available and to exclude compromised circulation as a variable. Selected study subjects were three healthy, young adult domestic dogs euthanized for behavioral reasons. The protocol for euthanasia recommended by the Florida Animal Control Association was followed in accordance with the American Veterinary Medical Association (AVMA) guidelines (Leary et al. 2013). The dogs were first sedated with an intramuscular injection of tiletamine-zolazepam, ketamine and xylazine (TKX) before intravenous injection of pentobarbital sodium (Fatal-Plus ${ }^{\circledast}$, Vortech Pharmaceuticals. Ltd., Dearborn, MI). Refer to Table 1 for individual dosing information. The cadavers were transported from the shelter to the University of Florida College of Veterinary Medicine, where they were stored at $2.2{ }^{\circ} \mathrm{C}$. Sample collection occurred within 48 hours of death. The sample collection and physical examination process took approximately 3 to 5 hours per animal.

The calibration standard solution contained a mixture of compounds used for an acid quantitation procedure designed for forensic casework and included pentobarbital purchased from Cerilliant Corporation (Round Rock, TX). Hexobarbital purchased from Sigma-Aldrich (St. Louis, MO) was used as an internal standard. Quality control standards containing pentobarbital were purchased from W.R. Grace \& Co. (Columbia, MD).

ACS grade ethyl acetate, glacial acetic acid, hexane, methanol, potassium hydroxide, and potassium phosphate monobasic were purchased from Fisher Scientific (Hampton, NH). The derivatizing agent trimethylanilinium hydroxide (TMAH) was purchased from Thermo Scientific (Waltham, MA). Deionized water was acquired from a Milli- ${ }^{\circledR}$ system by MilliporeSigma (Burlington, MA). Blank human blood was purchased from LifeSouth Community Blood Centers Inc. (Gainesville, FL).

\footnotetext{
1 William R. Maples Center for Forensic Medicine, College of Medicine, University of Florida, 4800 SW 35th Drive, Gainesville, FL 32608, USA

2 College of Veterinary Medicine, University of Florida, 2015 SW 16th Avenue, Gainesville, FL 32608, USA

Corresponding author: Jason Byrd, jhbyrd@ufl.edu, 352-294-4091

Received 20 Dec 2018; in revised form 22 April 2019; accepted 24 April 2019.
} 
TABLE 1-Physical characteristics and dosing information for three euthanized canine subjects

\begin{tabular}{|c|c|c|c|}
\hline & \multicolumn{3}{|c|}{ Canine subject } \\
\hline & D-01 & D-02 & D-03 \\
\hline Sex & $\mathrm{MN}$ & $\mathrm{MN}$ & $\mathrm{Ml}$ \\
\hline Weight (kg) & 19.2 & 34.3 & 25.5 \\
\hline BCS (1-9) & 3 & 5 & 4 \\
\hline \multicolumn{4}{|l|}{ TKX } \\
\hline volume (mL) & 0.7 & 0.9 & 1.0 \\
\hline ROA & IM, R \& L hind legs & IM, L hind leg & $\mathrm{IM}, \mathrm{R}$ hind leg \\
\hline \multicolumn{4}{|l|}{ Fatal-Plus (390 mg/mL) } \\
\hline volume (mL) & 5.0 & 8.0 & 8.0 \\
\hline ROA & IV, L cephalic v. & IV, R cephalic v. & IV, L cephalic v. \\
\hline $\begin{array}{l}\text { Pentobarbital sodium, } \\
\text { dose }(\mathrm{mg} / \mathrm{kg})\end{array}$ & 102 & 91 & 122 \\
\hline
\end{tabular}

Samples were extracted using United Chemical Technologies (Bristol, PA) ZSDAU020 Clean Screen ${ }^{\circledast}$ DAU extraction columns. Eluents were dried down on a Zymark Turbovap ${ }^{\circledR}$ (Hopkinton, MA). Derivatized extracts were run on an Agilent 6890 series gas chromatograph equipped with a nitrogen-phosphorus detector (Santa Clara, CA). Samples were loaded with an Agilent 7683 series automatic liquid sampler and injector. A Phenomenex (Torrance, CA) Zebron ZB-5 capillary gas chromatography column (30-m length, $0.25-\mathrm{mm}$ internal diameter, $0.10-\mu \mathrm{m}$ film thickness) was used to achieve separation. Analysis occurred on Agilent ChemStation with DrugQuant software.

\section{Acidic drug quantitation by GC-NPD}

The University of Florida Forensic Toxicology Laboratory's procedure for acidic drug quantitation by GC-NPD was followed for sample analysis (Goldberger 2015). Blank human blood was used to prepare calibrators, controls, and blank matrix solutions. Many samples were run at dilutions up to 50 times in order to fall within the linear dynamic range of the calibration curve (2.5-25 $\mathrm{mg} / \mathrm{L})$. Hexobarbital internal standard was added to all samples except the blanks. Phosphate buffer $(0.1 \mathrm{M}, \mathrm{pH} 6)$ was added to all samples to adjust their $\mathrm{pH}$ before centrifugation. Solid-phase extraction was used to isolate pentobarbital from the biological matrix. Columns were conditioned with washes of $3 \mathrm{~mL}$ methanol, $3 \mathrm{~mL}$ DI water, and $1 \mathrm{~mL}$ phosphate buffer without allowing the column to dry before samples were added. Samples were then loaded onto the columns, washed with $3 \mathrm{~mL}$ DI water and $1 \mathrm{~mL} 1.0 \mathrm{M}$ acetic acid, and allowed to dry before a final rinse with $2 \mathrm{~mL}$ hexane. Elution of analytes of interest was accomplished by passing $3 \mathrm{~mL}$ of ethyl acetate:hexane $(1: 1, \mathrm{v} / \mathrm{v})$ slowly through the column. Samples were evaporated to dryness under a stream of nitrogen in a Turbovap ${ }^{\oplus}$ with a water bath set to $40{ }^{\circ} \mathrm{C}$. They were reconstituted with $200 \mu \mathrm{L} 0.02 \mathrm{M}$ TMAH as a derivatizing agent and submitted for GC-NPD analysis.

Runs included a blank, a blank with an internal standard, five calibrators $(2.5,5.0,10,15,25 \mathrm{mg} / \mathrm{L})$, and intermittent quality control samples. The internal standard technique was used for analysis, allowing for quantitation of unknown samples by comparing the detector response for the drug versus the internal standard to a calibration curve of known concentrations.

\section{Results}

Measurable quantities of pentobarbital were present in all fluid and tissue samples tested (Table 2, Figure 1). The blood, kidneys, and lungs were areas of notably high concentration.

TABLE 2-Concentration ( $m g / L$ for fluids, $m g / k g$ for tissues) of pentobarbital present in samples examined via GC-NPD for three euthanized canine subjects

\begin{tabular}{llll}
\hline \multirow{2}{*}{ Sample type } & \multicolumn{3}{c}{ Canine subject } \\
\cline { 2 - 4 } aq-01 & D-02 & D-03 \\
\hline aqueous humor (left) & 14 & 34 & $79^{\mathrm{a}}$ \\
aqueous humor (right) & 13 & 33 & 43 \\
bile & 48 & 37 & 28 \\
blood (heart) & 825 & 356 & 261 \\
brain (forebrain) & 148 & 171 & 210 \\
kidney (left) & 485 & 629 & 525 \\
kidney (right) & 491 & 741 & 897 \\
liver (central left lateral lobe) & 165 & 236 & 15 \\
liver (peripheral left lateral lobe) & 171 & 104 & 19 \\
lungb (left caudal lobe) & positivec & positive & positive \\
lungb (right caudal lobe) & positive & positivec & positive \\
spleen & 120 & 36 & 20 \\
urine & 12 & 4.8 & 21 \\
vitreous humor (left) & 13 & 29 & 64 a \\
vitreous humor (right) & 15 & 30 & 42
\end{tabular}

GC-NPD, gas chromatography coupled with nitrogen-phosphorus detector. a. Contaminated with blood.

b. "Positive" indicates that concentration exceeded linear dynamic range of calibration curve following dilution.

c. Hemorrhage or blood congestion visible during necropsy.

Blood-to-liver ratios were calculated to compare distribution between the three dogs. Heart blood was compared to both peripheral and central liver samples. These values were 5.0, 1.5, and 17 for central liver samples and 4.8,3.4, and 14 for peripheral liver samples from D-01, D-02, and D-03, respectively.

\section{Discussion}

Following sedation with TKX, an overdose of pentobarbital circulates rapidly and pervasively prior to death in healthy dogs. While the distribution may be ubiquitous, it is also inconsistent based on these initial findings and their dissimilar blood-to-liver ratios. The central ratios had a mean of 7.8 with a standard deviation of 8.1, and the peripheral ratios had a mean of 7.4 with a standard deviation of 5.7. Higher concentrations were expected in organs with greater blood supply, such as the liver and spleen; however, concentrations were variable and ranged from 15 to 236 $\mathrm{mg} / \mathrm{kg}$ in these samples.

The small sample size and the lack of a control animal limit the conclusions that can be drawn from this data. In addition to 


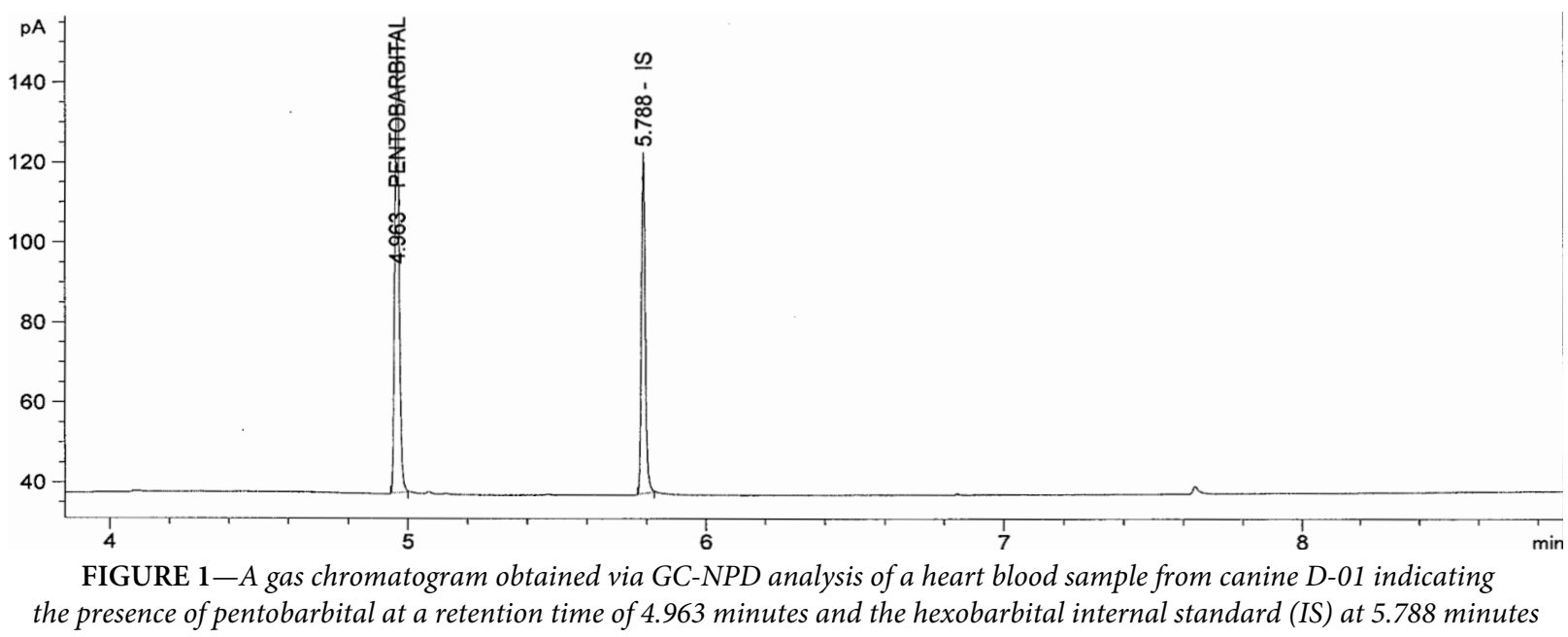

increasing the number of animals examined, several factors should be explored in future work. The study of pentobarbital distribution in bone is of interest. If present, pentobarbital is expected to persist, as this compound has been detected in soil up to two years after death in porcine decomposition research (Wyman et al. 2011). The validation of a standard additions technique for the analysis of matrices besides blood is needed to ensure that more reliable quantitative results are reported. Decomposition and postmortem redistribution may influence pentobarbital distribution, and a study that includes these components may better represent the quality and types of samples that are collected in the field. Age, disease states, the time between injection and death, various sedation and euthanasia methods, and species are also significant variables.

While detection of pentobarbital merely indicates that there was contact with this compound before the time of discovery, the reliable assessment of its presence or absence is useful to veterinarians. Toxicological results can corroborate or refute testimony in forensic casework. It can support or exclude pentobarbital overdose as a cause of death. However, accidental exposure or malicious poisonings may also yield positive results. This point is relevant to wildlife forensic work as improper disposal of euthanized remains poses a threat to protected species (Payne et al. 2012, Jurczynski and Zittlau 2007, Krueger and Krueger 2002). Efficient sampling and analysis protocols are needed so that resources are not exhausted by attempts to prove results are accurate. In this study, pentobarbital has been detected in traditional and non-traditional toxicological samples alike. Though further research is needed, both traditional and nontraditional biological samples may be tested for the presence of pentobarbital in cases of suspected euthanasia.

\section{Acknowledgments}

The authors thank the generous donors of the Elizabeth $\mathrm{R}$. Chapin Fund for their support, Jacksonville Animal Care \& Protective Services for donating the cadavers used for this research, and the personnel from the UF Forensic Toxicology Lab, the UF CA Pound Human Identification Lab, and the UF College of Veterinary Medicine for their ongoing guidance and assistance.

\section{Disclosures}

This research did not receive any specific grant from funding agencies in the public, commercial, or not-for-profit sectors.

\section{References}

Desrosiers NA, Watterson JH. 2010. The effect of burial on drug detection in skeletal tissues. Drug Test Anal. 2(7):346-56.

Goldberger BA. 2015. Acid quantitation by GC-NPD. University of Florida Forensic Toxicology Laboratory.

Jurczynski K, Zittlau E. 2007. Pentobarbital poisoning in Sumatran tigers (Panthera tigris sumatrae). J Zoo Wildl Med. 38(4):583-4.

Krueger B, Krueger BA. 2002. U.S. Fish and Wildlife Service fact sheet: secondary pentobarbital poisoning of wildlife. fws.gov/mountain-prairie/poison.pdf

Leary S, Underwood W, Anthony R, Cartner S, Corey D, Grandin T, Greenacre CB, Gwaltney-Bran S, Mccracken MA, Meyer R. 2013. AVMA guidelines for the euthanasia of animals, 2013 edition. American Veterinary Medical Association, Schaumburg, IL.

Nestlé Purina PetCare Center. 1999. Body condition system.

Payne J, Farris R, Parker G, Schwarz M, Bonhotal J. 2015. Quantification of sodium pentobarbital residues from equine mortality compost piles. J Anim Sci. 93(4):1824-9.

Watterson JH, Desrosiers NA, Betti CC, Dean D, Wyman JF. 2010. Relative distribution of drugs in decomposed skeletal tissue. J Anal Toxicol. 34(8):510-5.

Wyman JF, Dean DE, Yinger R, Simmons A, Broast D, Bissell M, Silveira F, Kelly N, Shott R, Ohr J, Howard R, Lewis B. 2011. The temporal fate of drugs in decomposing porcine tissue. J Forensic Sci. 56(3):694-9. 